(c) American Dairy Science Association, 2003.

\title{
Fractionation of Milk Fat by Short-Path Distillation
}

\author{
R. J. Campos, J. W. Litwinenko, and A. G. Marangoni \\ Dept. of Food Science \\ University of Guelph \\ Guelph, ON N1G2W1, Canada
}

\begin{abstract}
Fractionation of milk fat by short-path distillation changes the chemical composition and physical properties of the resulting fractions. Increases in distillation temperature from 125 to $250^{\circ} \mathrm{C}$ increased distillate yield from 0.3 to $42.7 \%$ (wt/wt). The distillate was enriched in short- and medium-chain fatty acids and low molecular weight acylglycerols, while the retentate was enriched in long-chain saturated and unsaturated fatty acids as well as high molecular weight acylglyerols. As distillation temperature increased, dropping points of the distillate increased. Relative to native milk fat, the solid fat content (SFC) vs. temperature melting profile of the distillate was depressed and that of the retentate was augmented, which correlated with the saturated long-chain fatty acid content in the fractions. Retentate crystallization parameters obtained by fitting the Avrami model to SFC-time data, did not change as a function of distillation temperature, but varied as a function of the degree of undercooling. Changes in microstructure observed by polarized light microscopy also appeared to be solely a function of the degree of undercooling, with no observable differences between retentates obtained at the different distillation temperatures. In addition, no changes in the retentate's free energy of nucleation $\left(\Delta \mathrm{G}_{\mathrm{c}}\right)$ as a function of distillation temperature were found. The compressive storage modulus of the crystallized retentate increased as a function of increasing distillation temperature.
\end{abstract}

(Key words: fractionation, milk fat, short-path distillation, crystallization)

Abbreviation key: $\mathbf{A G}=$ acylglycerol, $\Delta \mathbf{G}_{\mathbf{c}}=$ free energy of nucleation, $\mathbf{S F C}=$ solid fat contact, $\mathbf{T A G}=$ triacylgycerol.

\section{INTRODUCTION}

Milk fat is a very important commodity to the dairy industry. Its image as a natural product, and its organo-

Received June 7, 2002.

Accepted August 15, 2002.

Corresponding author: G. A. Marangoni; e-mail: amarango@ uoguelph.ca. leptic attributes, nutritional value, and functional properties make it suitable for numerous food applications (Boudreau and Arul, 1993; Rajah, 1994). Possessing a unique fatty acid profile, milk fat has the most complex chemical composition of all natural fats. This fatty acid composition is highly variable and is influenced by factors such as lactation stage, season, breed of cow, feed source, and region (Deffense, 1993; German and Dillard, 1998). More than 400 fatty acids, ranging from 4 to 28 carbon numbers, are present in milk fat (Jensen et al., 1991), of which approximately $70 \%$ are saturated fatty acids (Banks, 1991; German and Dillard, 1998). The two most abundant fatty acids in milk fat are the long-chain C16:0 and C18:1 (Banks, 1991). Appreciable amounts of shorter chain fatty acids (C4-C10) are also present (Nawar, 1996), contributing up to $10 \%$ of the fatty acid composition (Banks, 1991). Small amounts of branched and odd-numbered acids, dienes and trienes, hydroxy, and cyclic structures have also been identified (Nawar, 1996; Jimenez-Flores, 1997). With such an array of fatty acids, there is the potential to create up to 6 million different triacylglycerols (TAG) (JimenezFlores, 1997; van Aken and ten Grotenhuis, 1999). The distribution of fatty acids on the glycerol backbone of the TAG, however, is not random. Fatty acids are distributed in milk TAG asymmetrically with most of the short-chain fatty acids (C4-C10) at the sn-3 position (Jensen et al., 1991). A mixture of TAG with molecular weights ranging from 470 to 890 and 24 to 54 carbon numbers have been identified in milk fat (Boudreau and Arul, 1993).

The physical state and plastic properties of edible fats derive from both the molecular structure of the TAG and their crystal history. Milk fat is self-standing at room temperature and has a broad melting range between $-40^{\circ} \mathrm{C}$ and $40^{\circ} \mathrm{C}$ (Boudreau and Arul, 1993). The solid and liquid phases coexist and the solids melt gradually in this broad temperature range. These factors are essential to the functionality of milk fat as a plastic and spreadable fat (German and Dillard, 1998). Even with these unique physical characteristics, milk fat is not suitable for a number of food applications and has limited functionality.

Driven by the industrial demand to achieve a predictable and reproducible functionality, the separation of 
milk fat into various fractions with unique functional properties has been explored (Dimick et al., 1996). Fractionation involves the modification of the solid-liquid balance of fat (Hamm, 1995) based on differences in molecular weight, melting temperatures, volatility, and intermolecular interaction energy of the constituent TAG (Arul et al., 1988). Through the removal of a small quantity of high-melting (more saturated) TAG, milk fat is separated into a hard fraction or stearin and a liquid fraction or olein (Rajah, 1996; German and Dillard, 1998). The resulting fractions possess a narrower compositional range and, therefore, different physical properties (Hamm, 1995). The fatty acid composition of milk fat fractions is similar to that of native milk fat, yet some differences are found in the constituent TAG (German and Dillard, 1998). Heavier saturated TAG are almost completely removed from oleins, whereas they are concentrated in stearins. Lighter saturated TAG are reduced in stearins but remain unchanged in oleins. Monounsaturated TAG show the same trend, while diunsaturated TAG of high molecular weight are enriched in the oleins (Deffense, 1993). Differences in composition between fractions depend on how much liquid is entrained in the solid crystalline network, and the fact that short-chain fatty acids cannot be completely separated from long-chain fatty acids because they are attached to the same glycerol molecule (German and Dillard, 1998).

Different types of fractionation processes have been developed, which include melt, solvent, and detergent fractionation, supercritical fluid extraction and shortpath distillation. The most common process is cold or dry fractionation, in which the separation of TAG takes place on the basis of their melting points (Deffense, 1993; Dimick et al., 1996; Breitschuh, 1998; German and Dillard, 1998). Solvent (Boudreau and Arul, 1993; Breitschuh, 1998; Marangoni and Lencki, 1998; Hartel, 2001) and detergent fractionation (Hamm, 1995; Rajah, 1996) also takes place on the basis of melting points, but with the use of a solvent (i.e., acetone) or a surfactant solution to facilitate the separation of the fractions. In supercritical fluid extraction, separation is based on the solubility of the lipid species (Boudreau and Arul, 1993; Rajah, 1994; Hamm, 1995; Rizvi and Bhaskar, 1995), while in short-path distillation the separation takes place as a result of the volatility of the lipid constituents (Arul et al., 1998).

Short-path distillation involves the volatilization of molecules into a substantially gas-free space, e.g., vacuum. The controlling factor is the rate at which the molecules escape from the heated surface of the distilling liquid and are received by the cooled condenser surface. Contrary to other techniques, in short-path distillation there is a gradual increase in concentration of unsaturated long-chain fatty acids in the solid fraction (Arul et al., 1998). This technique also allows for the removal and isolation of vitamins, sterols, monoand diacylglycerols, fatty acids, and flavor compounds (Rahaj, 1994; Jimenez-Flores, 1997; Arul et al., 1998). Short-path distillation effects a very high degree of molecular weight separation, which offers an excellent opportunity to obtain fractions with distinctive chemical and physical properties without involving the addition of foreign compounds to the native milk fat (Arul et al., 1998).

The purpose of this work was to assess the potential of short-path distillation as a possible fractionation tool. A systematic study on the composition and physical properties of fractions obtained with this technology at different distillation temperatures is presented.

\section{METHODS AND MATERIALS}

\section{Fractionation Apparatus and Conditions}

Short-path distillation was carried out using a Pope 2-inch diameter laboratory wiped-film, short-path molecular still (Pope Scientific Inc., WI). An RV3 rotary vane pump connected in series to a Diffstak Mk2 diffusion pump (Edwards High Vacuum International, Manor Royal, UK) provided the required vacuum.

Before the actual distillation run, $500 \mathrm{~g}$ of molten milk fat were filtered through a Whatman \#1 filter paper in a heated Buchner funnel, and was then degassed by passing the molten fat through the Wiped-Film Still at $100^{\circ} \mathrm{C}$ at a pressure of 80 millitorr. Actual distillation runs were then performed at a constant flow rate of 9.8 $\mathrm{g} / \mathrm{min}$, at a pressure of 8 to 14 millitorr, and blade rotation speed of 20 on the dial. Under these conditions, fractionations were performed at temperatures of 100 , $125,150,175,180,185,190,195,200,225$, and $250^{\circ} \mathrm{C}$. The distillate and retentate were collected, weighed, and stored at $5^{\circ} \mathrm{C}$ for the duration of the study.

\section{Lipid Composition}

Acylglycerol (AG) composition, in terms of carbon number, was determined by gas-liquid chromatography using a Shimadzu GC-8A (Shimadzu Corp., Tokyo, Japan) and a flame-ionization detector operated at $360^{\circ} \mathrm{C}$ as previously described (Rousseau et al., 1996). Derivatization of the samples to fatty acid methyl esters was performed (Bannon et al., 1985) and fatty acid composition determined with a Shimadzu GC-8A gas-liquid chromatograph (Shimadzu Corporation, Tokyo, Japan). Fatty acid separation was carried out with a 1.5-m glass column packed with $10 \%$ Silar ACP on acid-washed 80 100 mesh Chromosorb W. The temperature program used was 60 to $210^{\circ} \mathrm{C}$ at $8^{\circ} \mathrm{C} / \mathrm{min}$. The injector port 
and detector were operating at $230^{\circ} \mathrm{C}$. Response factors were applied for fatty acid analysis as described by Bannon et al. (1985).

\section{Melting Behavior}

Dropping points were measured with a model FP83 Mettler DP apparatus (Mettler, Zurich, Switzerland). Fully melted samples $\left(80^{\circ} \mathrm{C}\right.$ for $\left.30 \mathrm{~min}\right)$ were introduced into prechilled sample holders and held at $-10^{\circ} \mathrm{C}$ for $1 \mathrm{~h}$ before measurements. A heating rate of $1^{\circ} \mathrm{C} / \mathrm{min}$ was employed.

Solid fat content (SFC) was measured by pulsed nuclear magnetic resonance with a Bruker PC20 Series NMR analyzer (Bruker, Milton, ON, Canada) according to AOCS official method Cd 16-81 (AOCS, 1983). All retentate samples were held at $80^{\circ} \mathrm{C}$ for $30 \mathrm{~min}$ before analysis to eliminate crystal history.

\section{Kinetics of Crystallization}

Crystallization kinetics were characterized at 5, 10, $15,20,23$, and $25^{\circ} \mathrm{C}$. Retentate samples acquired at each distillation temperature were melted in NMR tubes at $80^{\circ} \mathrm{C}$ for $30 \mathrm{~min}$. Crystallization curves were obtained at each of the aforementioned temperatures by placing the tubes in water baths and taking SFC readings at appropriate time intervals. The crystallization curves were fitted to the Avrami equation by least squares nonlinear regression (Marangoni, 1998) using GraphPad Prizm 3.0 (GraphPad Software Incorporated, San Diego, CA). The Avrami equation (Avrami, 1939) is applied to fat systems as:

$$
\frac{S F C(t)}{S F C_{\infty}}=1-e^{-k t^{\mathrm{n}}}
$$

where $S F C(t)$ and $S F C_{\infty}$ are the SFC (\%) at time $t$ and the maximum SFC after crystallization was completed, respectively. Fitting the SFC data as a function of time to this model allows for the determination the Avrami parameters, which provide information on the nature of the crystallization process. The Avrami constant (k), represents the crystallization rate constant. The Avrami exponent or index of crystallization ( $n$ ), indicates the crystal growth mechanism. This index is a combined function of time dependence of nucleation and the number of dimensions in which growth takes place (Sharples, 1966; Wright et al., 2000).

Induction times $(\tau)$ of crystallization were determined by extrapolating from the linearly increasing portion of the SFC curve to the time axis. Half-times of crystallization that reflect the magnitudes of the rate constants were also used to characterize crystallization kinetics. They were calculated using the following relationship:

$$
t_{1 / 2}=\left(\frac{0.693}{k}\right)^{1 / n}
$$

Using $\tau$, the apparent free energies of nucleation $\left(\Delta \mathrm{G}_{\mathrm{c}}\right)$ were determined at each temperature. $\Delta \mathrm{G}_{\mathrm{c}}$ depends on the degree of undercooling, which can influence polymorphic form and microstructure. With sufficient undercooling, a melt becomes supersaturated and nucleation occurs resulting in a lowering of the overall free energy of the system. The free energy of nucleation was calculated using the Fisher-Turnbull and GibbsThompson equations as described in previous studies (Wright et al., 2000).

\section{Microstructure}

A small droplet (about $10 \mu \mathrm{l}$ ) of melted retentate sample with crystal history erased was placed on a preheated (at $80^{\circ} \mathrm{C}$ ) glass slide, using a preheated capillary tube. A preheated glass coverslip was carefully placed over the sample to produce a film of uniform thickness. The slides were then placed directly into incubators and allowed to crystallize statically at temperatures of 5,21 , and $25^{\circ} \mathrm{C}$. Following storage for $24 \mathrm{~h}$, samples were imaged on a temperature-controlled microscope stage (Linkam Scientific Instruments, Surrey, UK). When viewed by polarized light microscopy on an Olympus BX60 light microscope (Olympus America Ltd., Melville, NY) the birefringent solid microstructural elements of the network could be directly observed. Digital images were acquired via a black and white SenSys array camera (Photometrics Ltd., Trenton, NJ) and PCI video capture board (Photometrics Ltd.). From these images, qualitative observations about the resulting crystal network could be made.

\section{Rheology}

Small deformation rheological testing was performed using a DMA7 dynamic mechanical analyzer (Perkin Elmer, Wellesley, MA) with a $10-\mathrm{mm}$ diameter parallel plate geometry. Retentate samples acquired at each distillation temperature were melted to erase crystal history, then transferred into molds prechilled to $5^{\circ} \mathrm{C}$ producing cylindrical test samples $10 \mathrm{~mm}$ in diameter and $6 \mathrm{~mm}$ in height. These samples were stored at $5^{\circ} \mathrm{C}$ for $24 \mathrm{~h}$ before analysis and analyzed at $5^{\circ} \mathrm{C}$. Dynamic stress sweeps were carried out from 1000 to $10,000 \mathrm{~Pa}$ with a static force of $1000 \mathrm{~Pa}$ at a frequency of $1 \mathrm{~Hz}$. The compressive storage modulus $\left(\mathrm{E}^{\prime}\right)$ was determined from the linear viscoelastic region.

\section{RESULTS AND DISCUSSION}

Milk fat was fractionated by short-path distillation at different fractionation temperatures yielding a distil- 


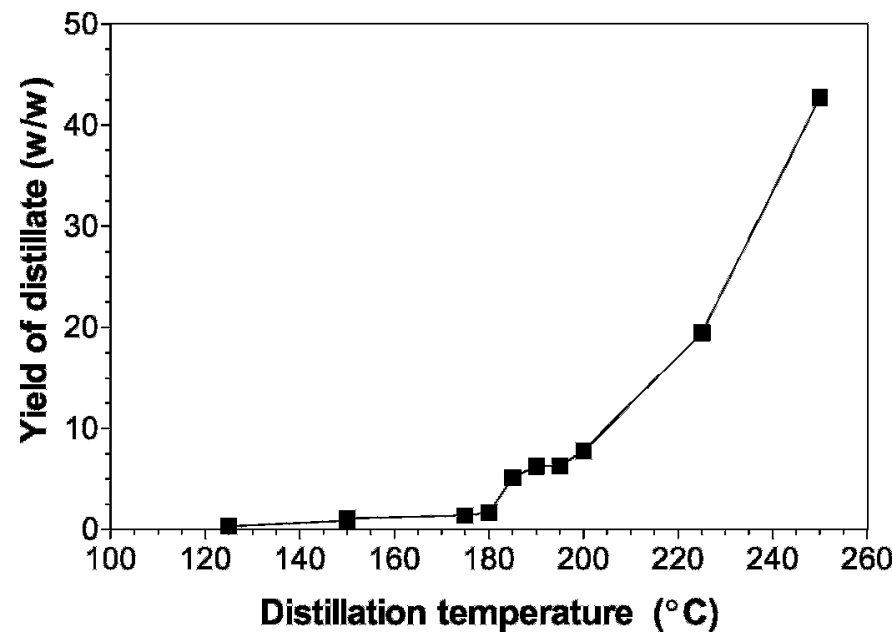

Figure 1. Yield $(\mathrm{w} / \mathrm{w})$ of distillate obtained at various distillation temperatures.

late and a residual retentate. Distillate yields obtained under the different distillation conditions are shown in Figure 1. No separation was obtained at $100^{\circ} \mathrm{C}, 125^{\circ} \mathrm{C}$ being the minimum temperature at which fractionation was evident. Distillate yield increased exponentially from 0.3 to $42.7 \%$ in the temperature range 125 to $250^{\circ} \mathrm{C}$.

\section{Chemical Composition}

The fatty acid profile of the distillate and retentate obtained at different fractionation temperatures, along with that of native milk fat, are reported in Tables 1 and 2. A higher concentration of short (C4 to $\mathrm{C} 8$ ) and medium-chain fatty acids (C10 to C15), and a lower concentration of long-chain fatty acids (C16 to $\mathrm{C} 22)$ was observed in the distillate relative to native milk fat as shown in Figure 2. The opposite trend was observed in the retentate, although the effects were not as dramatic. At all fractionation temperatures, the retentate was enriched in unsaturated fatty acids and depleted in saturated fatty acids relative to the distillate. The observed trends in fatty acid composition of the fractions agree with those reported by other authors in that higher melting fractions obtained by other fractionation methods, such as dry and solvent fractionation, are depleted in short-chain fatty acids and enriched in longchain fatty acids (Deffense, 1993; Breitschuh, 1998; van Aken et al., 1999). However, in contrast to other fractionation processes, the level of unsaturated fatty acids in the heavy fraction (retentate) obtained by this fractionation technology is higher than in the distillate (light fraction) as reported by Arul et al. (1988). This highlights the differences in the nature of the separation process.

The AG composition (acyl carbon numbers) of both the retentate and the distillate obtained at different fractionation temperatures are presented in Tables 3 and 4. At all fractionation temperatures, the distillate had a higher concentration of low molecular weight AG (C18 to C34) than the retentate. The opposite was observed for high molecular weight AG (C42 to C54). The retentate had a higher concentration of high molecular weight AG than the distillate at all temperatures. The distillation temperature was found to affect the AG composition of the resulting fractions. At $125^{\circ} \mathrm{C}$, over $80 \%$ of the AG present in the distillate are of low molecular weight (C18 to C34), including some that are absent in the retentate (C18 to $\mathrm{C} 22$ ). Figure 3 shows that as the fractionation temperature increases, the concentration of low molecular weight $\mathrm{AG}$ (C18 to $\mathrm{C} 34)$ in the

Table 1. Fatty acid composition (\% area) of native milk fat and distillate obtained by short-path distillation at different distillation temperatures.

\begin{tabular}{|c|c|c|c|c|c|c|c|c|c|c|c|}
\hline \multirow{2}{*}{$\begin{array}{l}\text { Fatty } \\
\text { acid }\end{array}$} & \multirow{2}{*}{$\begin{array}{l}\text { Milk } \\
\text { fat }\end{array}$} & \multicolumn{10}{|c|}{ Distillation temperature $\left({ }^{\circ} \mathrm{C}\right)$} \\
\hline & & 125 & 150 & 175 & 180 & 185 & 190 & 195 & 200 & 225 & 250 \\
\hline $4: 0$ & 3.3 & 4.4 & 10.7 & 7.7 & 12.5 & 9.5 & 9.7 & 7.5 & 9.0 & 8.1 & 4.8 \\
\hline $6: 0$ & 2.6 & 3.1 & 7.1 & 4.7 & 7.4 & 5.3 & 5.7 & 4.6 & 5.1 & 4.8 & 3.4 \\
\hline 8:0 & 1.5 & 2.2 & 5.6 & 3.5 & 5.5 & 3.7 & 4.1 & 0.1 & 3.6 & 2.9 & 2.0 \\
\hline $10: 0$ & 3.3 & 4.6 & 9.3 & 6.8 & 9.4 & 7.1 & 7.6 & 7.9 & 7.0 & 5.5 & 4.0 \\
\hline $12: 0$ & 3.5 & 5.1 & 8.0 & 6.3 & 8.2 & 7.4 & 0.1 & 8.3 & 7.0 & 5.7 & 4.3 \\
\hline 14:0 & 10.8 & 13.0 & 15.1 & 14.1 & 14.3 & 16.3 & 16.9 & 17.8 & 16.0 & 14.4 & 12.4 \\
\hline $14: 1$ & 1.8 & 2.1 & 1.7 & 1.9 & 1.7 & 1.8 & 2.0 & 2.1 & 1.8 & 1.9 & 1.9 \\
\hline $15: 0$ & 1.3 & 1.6 & 1.5 & 1.6 & 1.7 & 1.6 & 1.8 & 1.7 & 1.6 & 1.4 & 1.4 \\
\hline $16: 0$ & 27.6 & 25.2 & 21.6 & 24.4 & 21.7 & 28.7 & 30.7 & 30.3 & 26.7 & 27.8 & 28.8 \\
\hline $16: 1$ & 2.9 & 2.8 & 2.0 & 2.4 & 1.9 & 2.2 & 2.4 & 2.3 & 2.3 & 2.6 & 2.8 \\
\hline 18:0 & 11.0 & 8.5 & 4.1 & 6.7 & 4.0 & 4.8 & 5.6 & 5.1 & 5.5 & 6.7 & 9.1 \\
\hline 18:1 & 25.1 & 20.7 & 10.5 & 15.7 & 9.1 & 9.9 & 11.5 & 10.5 & 12.0 & 15.3 & 20.5 \\
\hline 18:2 & 3.2 & 2.9 & 1.6 & 2.1 & 1.4 & 1.0 & 1.2 & 1.1 & 1.5 & 1.9 & 2.7 \\
\hline 18:3 & 0.8 & 1.8 & 0.7 & 1.4 & 0.8 & 0.4 & 0.5 & 0.4 & 0.5 & 0.5 & 1.0 \\
\hline $20: 0$ & 1.1 & 2.0 & 0.4 & 0.7 & 0.3 & 0.3 & 0.3 & 0.3 & 0.5 & 0.4 & 1.0 \\
\hline
\end{tabular}


Table 2. Fatty acid composition (\% area) of native milk fat and retentate obtained by short-path distillation at different distillation temperatures.

\begin{tabular}{|c|c|c|c|c|c|c|c|c|c|c|c|}
\hline \multirow{2}{*}{$\begin{array}{l}\text { Fatty } \\
\text { acid }\end{array}$} & \multirow{2}{*}{$\begin{array}{l}\text { Milk } \\
\text { fat }\end{array}$} & \multicolumn{10}{|c|}{ Distillation temperature $\left({ }^{\circ} \mathrm{C}\right)$} \\
\hline & & 125 & 150 & 175 & 180 & 185 & 190 & 195 & 200 & 225 & 250 \\
\hline $4: 0$ & 3.3 & 3.3 & 3.3 & 2.4 & 4.4 & 1.8 & 3.1 & 2.5 & 3.0 & 2.0 & 1.0 \\
\hline $6: 0$ & 2.6 & 2.5 & 2.5 & 1.9 & 3.3 & 2.0 & 2.4 & 2.1 & 2.3 & 2.0 & 1.3 \\
\hline $8: 0$ & 1.5 & 1.5 & 1.5 & 1.2 & 2.0 & 1.3 & 1.4 & 1.4 & 1.4 & 1.3 & 1.0 \\
\hline $10: 0$ & 3.3 & 3.3 & 3.2 & 2.8 & 4.2 & 3.1 & 3.2 & 3.1 & 3.1 & 2.9 & 2.7 \\
\hline $12: 0$ & 3.5 & 3.6 & 3.5 & 3.3 & 4.5 & 3.8 & 3.9 & 3.8 & 3.4 & 3.1 & 3.1 \\
\hline 14:0 & 10.8 & 11.0 & 10.8 & 10.7 & 12.3 & 11.9 & 11.9 & 11.9 & 10.7 & 10.0 & 10.2 \\
\hline $14: 1$ & 1.8 & 1.8 & 1.8 & 1.8 & 2.0 & 2.0 & 2.0 & 2.0 & 1.8 & 1.8 & 1.8 \\
\hline $15: 0$ & 1.3 & 1.2 & 1.2 & 1.3 & 1.3 & 1.4 & 1.4 & 1.4 & 1.2 & 1.2 & 1.2 \\
\hline $16: 0$ & 27.6 & 28.0 & 27.8 & 28.0 & 30.6 & 32.7 & 31.8 & 32.4 & 27.8 & 26.9 & 27.3 \\
\hline $16: 1$ & 2.9 & 2.9 & 2.9 & 3.0 & 2.7 & 3.0 & 2.9 & 2.9 & 3.0 & 3.1 & 3.1 \\
\hline 18:0 & 11.0 & 10.9 & 11.0 & 11.4 & 9.3 & 10.7 & 10.0 & 10.5 & 11.4 & 11.9 & 12.4 \\
\hline 18:1 & 25.1 & 25.2 & 25.7 & 26.0 & 20.0 & 22.6 & 22.1 & 22.3 & 26.3 & 28.1 & 29.0 \\
\hline $18: 2$ & 3.2 & 3.1 & 3.2 & 3.6 & 2.0 & 2.3 & 2.3 & 2.3 & 3.2 & 3.6 & 3.6 \\
\hline 18:3 & 0.8 & 0.8 & 0.9 & 1.2 & 0.6 & 0.7 & 0.7 & 0.7 & 0.8 & 1.0 & 0.9 \\
\hline $20: 0$ & 1.1 & 0.9 & 0.8 & 1.4 & 0.7 & 0.9 & 0.8 & 0.9 & 0.9 & 1.1 & 1.2 \\
\hline
\end{tabular}

distillate decreases significantly until they account for less than $20 \%$ of the total $\mathrm{AG}$ at $250^{\circ} \mathrm{C}$. The concentration of medium- (C36 to C40) and high- (C42 to C54) molecular weight AG decreases in distillates obtained at fractionation temperatures up to $150^{\circ} \mathrm{C}$, followed by an enrichment at higher temperatures. The AG composition of the retentate was also affected by the distillation temperature, although not as dramatically as the distillate. The retentate was enriched in high molecular weight AG (C42 to C54) and depleted in medium- (C36 to $\mathrm{C} 40$ ) and low molecular weight AG (C24 to C34) as the distillation temperature increased. At fractionation temperatures of $175^{\circ} \mathrm{C}$ and higher, 54-carbon AG became evident in the retentate. Under our analytical conditions, these AG were not detected in the retentate at lower distillation temperatures.

\section{Melting Behavior}

As reported by other research groups, fractionation processes render a heavy fraction with high melting point relative to that of native milk fat, and a light fraction with a low melting point (Deffense, 1993; Krishnamurthy and Kellens, 1996; Arul et al., 1998). The thermal properties of the resulting fractions, measured in terms of dropping point, are shown in Figure 4. A slight increase in the dropping point of the samples was observed as the distillation temperature increased. The dropping point of the distillate is about $16^{\circ} \mathrm{C}$ for fractions distilled below $225^{\circ} \mathrm{C}$. At $250^{\circ} \mathrm{C}$, a major increase to $27.9^{\circ} \mathrm{C}$ was observed. The retentate, on the other hand, had higher dropping points (roughly $35^{\circ} \mathrm{C}$ ) at all fractionation temperatures.

Figure 5A shows the melting profile of distillates obtained at different fractionation temperatures as well as that of native milk fat. The melting profile of the distillate approaches that of native milk fat as the distillation temperature is increased. In general, the SFC of distillates are lower than those of native milk fat in the temperature range 0 to $40^{\circ} \mathrm{C}$. As seen on Figure $5 \mathrm{~B}$, the $\mathrm{SFC}$ of the retentates are higher than that of
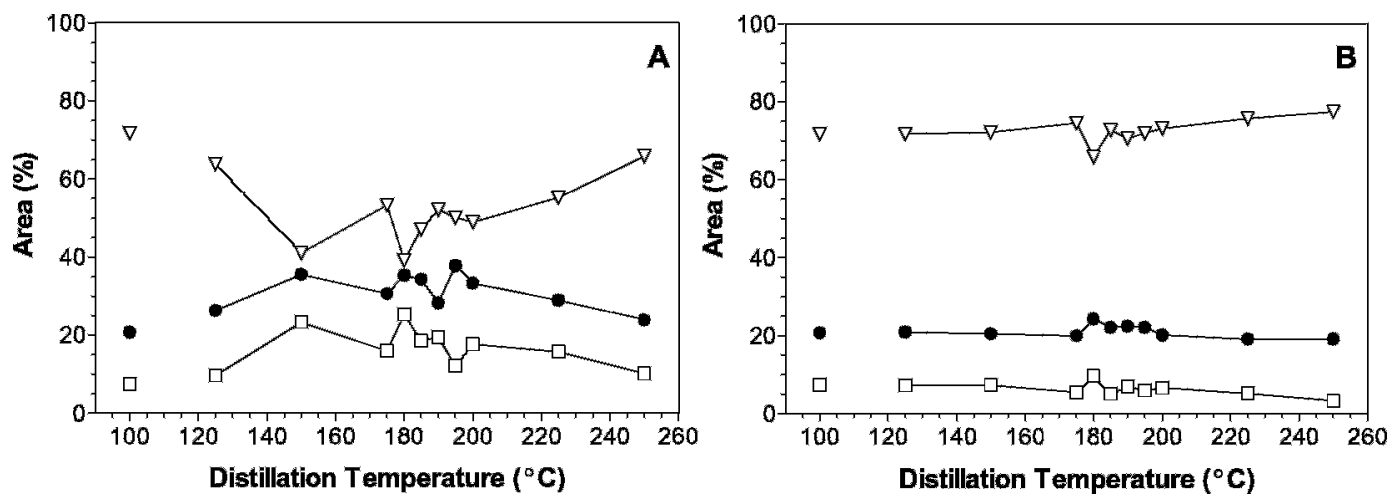

Figure 2. Fatty acid composition (area \%) of the distillate (A) and retentate (B). Short-chain fatty acids (C4 to C8) $\square$, medium-chain fatty acids (C10 to C15) long-chain fatty acids (C16 to C20) $\nabla$. Symbols at $100^{\circ} \mathrm{C}$ correspond to the composition of native milk fat. 
Table 3. Acylglycerol composition (\% area) of native milk fat and distillate obtained by short-path distillation at different distillation temperatures.

\begin{tabular}{|c|c|c|c|c|c|c|c|c|c|c|c|}
\hline \multirow{2}{*}{$\begin{array}{l}\text { Acyl } \\
\text { carbon } \\
\text { number }^{1}\end{array}$} & \multirow{2}{*}{$\begin{array}{l}\text { Milk } \\
\text { fat }\end{array}$} & \multicolumn{10}{|c|}{ Temperature of fractionation $\left({ }^{\circ} \mathrm{C}\right)$} \\
\hline & & 125 & 150 & 175 & 180 & 185 & 190 & 195 & 200 & 225 & 250 \\
\hline 18 & 0.0 & 28.6 & 0.0 & 5.2 & 6.1 & 0.6 & 0.0 & 1.2 & 0.0 & 0.0 & 0.0 \\
\hline 20 & 0.0 & 33.2 & 3.7 & 8.6 & 0.0 & 1.3 & 0.0 & 2.3 & 1.0 & 0.0 & 0.0 \\
\hline 21 & 0.0 & 2.6 & 6.0 & 1.9 & 2.8 & 0.5 & 0.8 & 0.7 & 0.3 & 0.0 & 0.0 \\
\hline 22 & 0.0 & 1.6 & 8.2 & 2.5 & 3.9 & 1.3 & 1.9 & 1.1 & 1.1 & 0.7 & 0.3 \\
\hline 24 & 0.7 & 1.5 & 38.7 & 4.4 & 4.7 & 1.8 & 2.6 & 1.3 & 1.6 & 1.0 & 0.4 \\
\hline 26 & 0.7 & 8.1 & 12.9 & 23.1 & 23.2 & 10.0 & 13.7 & 6.6 & 8.0 & 3.4 & 1.4 \\
\hline 28 & 1.3 & 1.6 & 9.3 & 9.5 & 10.8 & 7.8 & 8.9 & 5.5 & 7.1 & 3.2 & 1.4 \\
\hline 30 & 2.4 & 1.3 & 7.3 & 7.9 & 10.1 & 10.2 & 9.8 & 7.7 & 10.1 & 5.2 & 2.3 \\
\hline 32 & 4.9 & 1.4 & 5.8 & 6.9 & 9.9 & 13.4 & 11.1 & 11.5 & 13.5 & 8.7 & 4.4 \\
\hline 34 & 10.0 & 2.1 & 4.5 & 6.4 & 10.3 & 17.1 & 13.9 & 15.7 & 17.1 & 15.0 & 9.1 \\
\hline 36 & 13.8 & 3.3 & 2.7 & 6.7 & 9.3 & 18.3 & 16.3 & 19.3 & 18.8 & 21.3 & 16.0 \\
\hline 38 & 11.2 & 3.9 & 0.9 & 5.6 & 5.2 & 10.4 & 11.2 & 13.1 & 11.9 & 20.5 & 19.5 \\
\hline 40 & 7.3 & 3.3 & 0.0 & 3.5 & 2.1 & 3.9 & 5.2 & 6.0 & 5.0 & 11.1 & 13.7 \\
\hline 42 & 6.5 & 1.8 & 0.0 & 1.7 & 0.8 & 1.4 & 2.2 & 2.6 & 1.8 & 4.1 & 7.2 \\
\hline 44 & 7.5 & 1.4 & 0.0 & 1.3 & 0.4 & 0.8 & 1.2 & 1.7 & 1.0 & 2.2 & 5.4 \\
\hline 46 & 10.5 & 1.3 & 0.0 & 1.4 & 0.2 & 0.6 & 0.7 & 1.5 & 0.8 & 1.4 & 5.1 \\
\hline 48 & 11.9 & 1.5 & 0.0 & 1.5 & 0.0 & 0.5 & 0.5 & 1.5 & 0.7 & 1.3 & 5.4 \\
\hline 50 & 8.7 & 1.5 & 0.0 & 1.4 & 0.0 & 0.0 & 0.0 & 0.7 & 0.3 & 0.8 & 5.4 \\
\hline 52 & 2.4 & 0.0 & 0.0 & 0.7 & 0.0 & 0.0 & 0.0 & 0.0 & 0.0 & 0.0 & 2.9 \\
\hline
\end{tabular}

${ }^{1}$ Excluding glycerol.

native milk fat in the temperature range of $0^{\circ} \mathrm{C}$ to $40^{\circ} \mathrm{C}$. Generally, in the temperature range $0^{\circ} \mathrm{C}$ to $20^{\circ} \mathrm{C}$, there is a significant positive correlation $(P \leq 0.05)$ between the concentration of saturated long-chain fatty acids (C16:0, C18:0, and C20:0) in retentates obtained under different distillation temperatures and their SFC; as well as between the distillation temperatures and the $\mathrm{SFC}$ of the retentates $(P \leq 0.05)$ obtained in the temperature range of 15 to $35^{\circ} \mathrm{C}$. Such correlations explain the increase in solid crystalline material as a function of distillation temperature. At higher crystallization temperatures, the quantity of crystalline mass is reduced, as the system approaches its melting point (as shown in Figure 4); hence, the correlations become nonsignificant. The observed trends correspond to those reported for high melting stearins and low melting oleins obtained by other fractionation methods (Kaylegian and Lindsay, 1992; Deffense, 1993; Krishnamurthy and Kellens, 1996; Dimick et al., 1996) as well as to the distillates and retentates obtained by short-path distillation under different operation conditions (Arul et al., 1988).

Table 4. Acylglycerol composition (\% area) of native milk fat and retentate obtained by short-path distillation at different distillation temperatures.

\begin{tabular}{|c|c|c|c|c|c|c|c|c|c|c|c|}
\hline \multirow{2}{*}{$\begin{array}{l}\text { Acyl } \\
\text { carbon } \\
\text { number }^{1}\end{array}$} & \multirow{2}{*}{$\begin{array}{l}\text { Milk } \\
\text { fat }\end{array}$} & \multicolumn{10}{|c|}{ Fractionation temperature $\left({ }^{\circ} \mathrm{C}\right)$} \\
\hline & & 125 & 150 & 175 & 180 & 185 & 190 & 195 & 200 & 225 & 250 \\
\hline 24 & 0.7 & 0.2 & 0.3 & 0.0 & 0.2 & 0.0 & 0.0 & 0.0 & 0.0 & 0.0 & 0.0 \\
\hline 26 & 0.7 & 0.7 & 0.5 & 0.5 & 0.5 & 0.0 & 0.2 & 0.3 & 0.0 & 0.0 & 0.0 \\
\hline 28 & 1.3 & 0.7 & 1.0 & 0.6 & 0.5 & 0.2 & 0.4 & 0.4 & 0.0 & 0.0 & 0.0 \\
\hline 30 & 2.4 & 1.2 & 2.0 & 1.1 & 1.1 & 0.6 & 0.9 & 0.8 & 0.4 & 0.0 & 0.0 \\
\hline 32 & 4.9 & 2.2 & 4.8 & 2.0 & 2.5 & 1.6 & 2.1 & 1.9 & 1.3 & 0.6 & 0.6 \\
\hline 34 & 10.0 & 5.2 & 10.7 & 4.8 & 5.9 & 5.3 & 5.6 & 5.1 & 3.9 & 2.2 & 1.8 \\
\hline 36 & 13.8 & 10.4 & 13.4 & 9.5 & 12.1 & 11.1 & 11.1 & 11.7 & 9.3 & 6.3 & 5.1 \\
\hline 38 & 11.2 & 14.2 & 11.1 & 13.2 & 13.6 & 13.1 & 13.9 & 13.6 & 14.1 & 11.3 & 9.1 \\
\hline 40 & 7.3 & 11.6 & 7.7 & 11.0 & 10.4 & 10.2 & 10.8 & 10.7 & 12.0 & 11.1 & 9.3 \\
\hline 42 & 6.5 & 7.5 & 6.6 & 7.0 & 7.3 & 8.0 & 7.6 & 7.5 & 7.8 & 7.7 & 7.0 \\
\hline 44 & 7.5 & 7.0 & 7.4 & 6.6 & 7.0 & 8.1 & 7.3 & 7.3 & 7.3 & 7.8 & 7.6 \\
\hline 46 & 10.5 & 8.0 & 10.2 & 7.6 & 7.7 & 9.3 & 8.0 & 8.1 & 8.4 & 9.3 & 9.6 \\
\hline 48 & 11.9 & 10.1 & 11.7 & 10.7 & 10.4 & 10.9 & 10.7 & 10.8 & 10.1 & 11.4 & 12.3 \\
\hline 50 & 8.7 & 12.3 & 9.3 & 12.2 & 10.9 & 11.4 & 11.3 & 11.5 & 13.0 & 15.2 & 17.2 \\
\hline 52 & 2.4 & 8.8 & 3.1 & 9.7 & 7.6 & 7.8 & 7.8 & 7.9 & 9.7 & 12.5 & 14.5 \\
\hline 54 & 0.0 & 0.0 & 0.0 & 3.5 & 2.3 & 2.2 & 2.1 & 2.3 & 2.6 & 4.6 & 5.7 \\
\hline
\end{tabular}

${ }^{1}$ Excluding glycerol. 

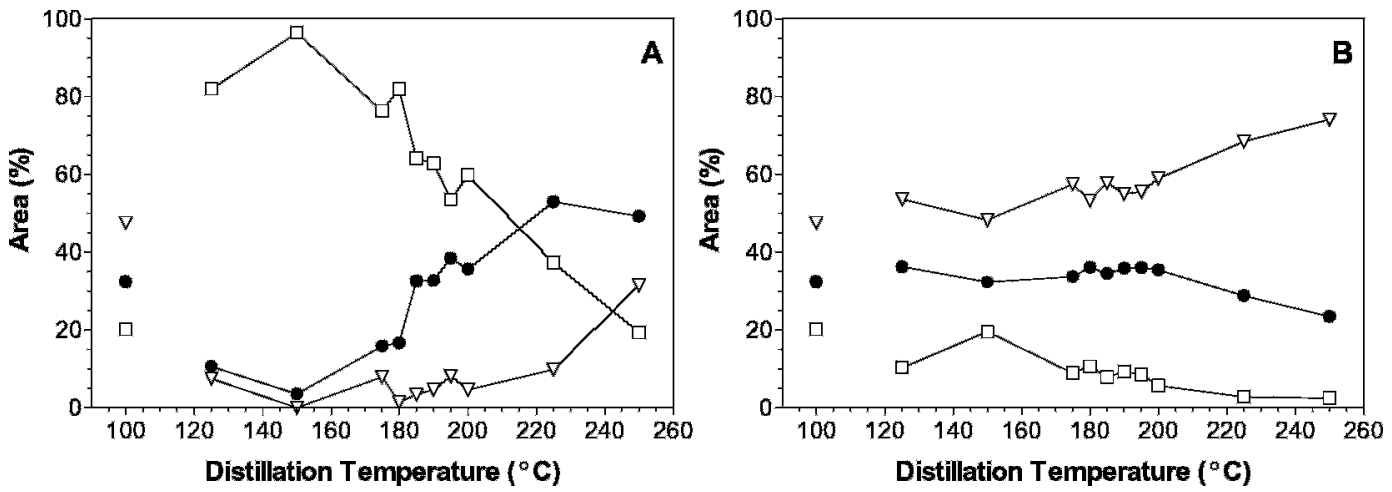

Figure 3. Acylglycerol composition (area \%) of the distillate (A) and retentate (B). Low molecular weight triacylglycerol (TAG) (C18 to C34) $\square$, medium molecular weight TAG (C36 to C40) $\bullet$, high molecular weight TAG (C42 to C54) $\nabla$. Symbols at $100^{\circ} \mathrm{C}$ correspond to the composition of native milk fat.

\section{Crystallization Kinetics}

The quantification of the crystallization behavior of retentates obtained at different distillation temperatures was obtained by means of measurement of the development of crystalline material as a function of time was measured. The resulting SFC vs. time crystallization curves obtained at different crystallization temperatures, shown in Figure 6, were fitted to the Avrami equation (Avrami, 1939). The Avrami constants (k), Avrami exponents $(n)$, and half times of crystallization $\left(t_{1 / 2}\right)$ as a function of crystallization temperature are reported in Figure 7.

The existence of two regions of distinct crystallization behavior is demonstrated in Figure 7 , one below $20^{\circ} \mathrm{C}$, where the values of $\mathrm{k}$ are high whereas values for $n$ and $t_{1 / 2}$ are low; and the other above $20^{\circ} \mathrm{C}$ where a

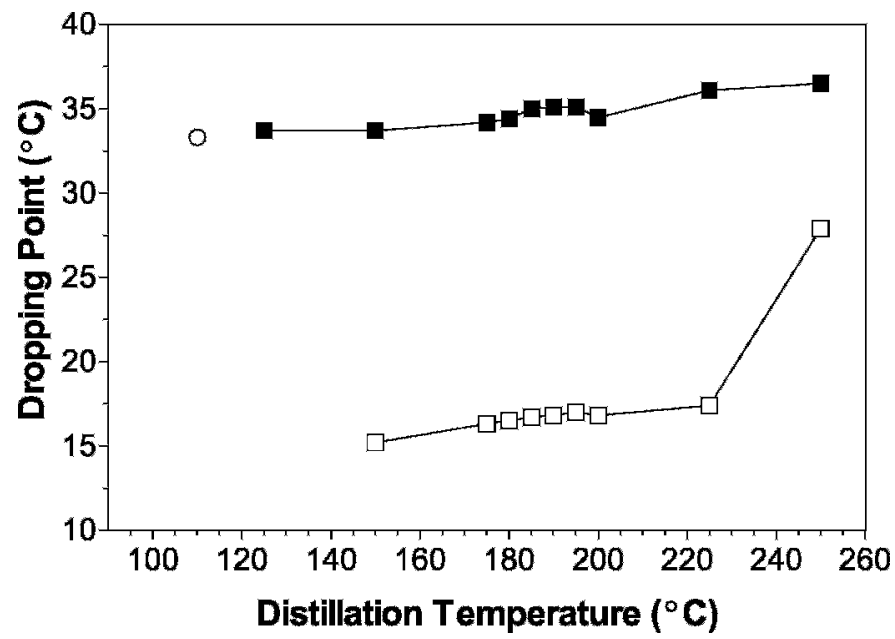

Figure 4. Dropping points $\left({ }^{\circ} \mathrm{C}\right)$ of distillate $(\square)$, retentate $(\square)$, and native milk fat $(O)$ at different fracctionation temperatures $\left({ }^{\circ} \mathrm{C}\right)$.
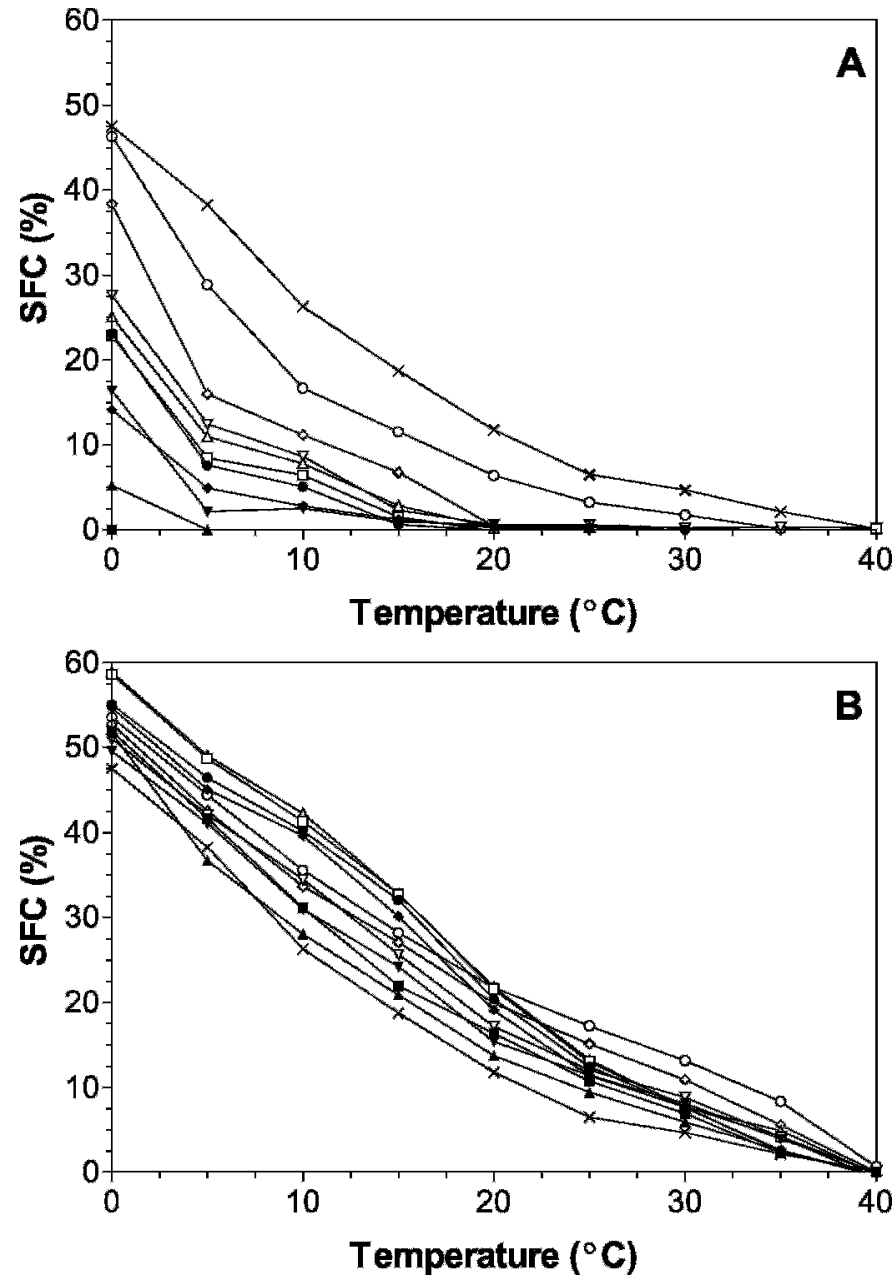

Figure 5. Solid fat content $(\%)$ vs. temperature $\left({ }^{\circ} \mathrm{C}\right)$ for the distillates (A) and retentates (B) obtained at $125(\boldsymbol{\square}), 150(\mathbf{\Delta}), 175(\boldsymbol{\nabla})$, $180(\diamond), 185(\bullet), 190(\square), 195(\triangle), 200(\nabla), 225(\diamond), 250^{\circ} \mathrm{C}(\bigcirc)$, and native milk fat $(\times)$. 
dramatic decrease in the value of $\mathrm{k}$ and augmentation in the values of $n$ and $t_{1 / 2}$ are observed. No trend on the crystallization parameters under the determined crystallization temperatures was found between the retentates obtained through different distillation conditions. As the crystallization temperature is increased, a decrease in the crystallization rates was observed (Figure 7A), with significantly higher crystallization indices (Figure 7B) and half-times of crystallization (Figure 7C), as well as more sigmoidal crystallization curves for all distillation temperatures. The crystallization parameters $\left(\mathrm{k}, n\right.$, and $\left.\mathrm{t}_{1 / 2}\right)$ are solely dependent on the degree of undercooling $(\Delta \mathrm{T})$, which determines the crystallization kinetics of the different fractions.
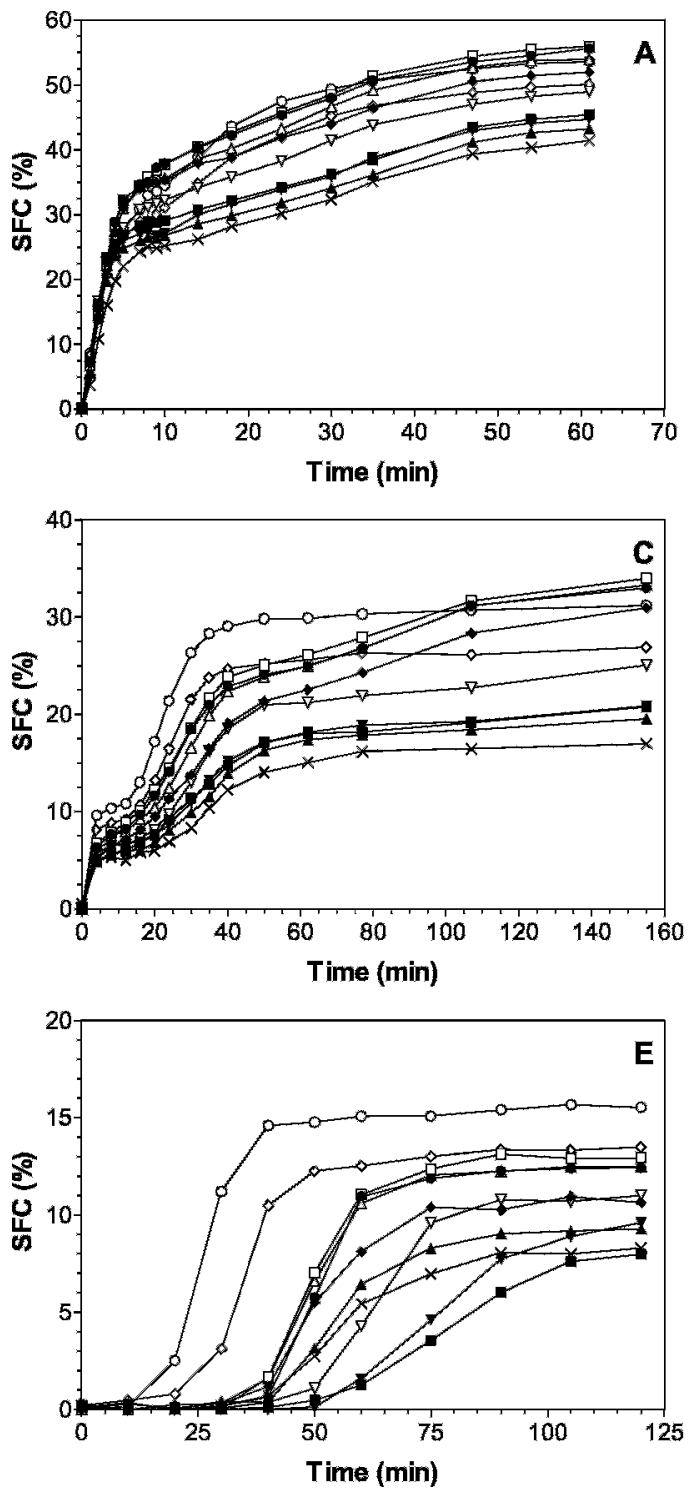

The activation $\Delta \mathrm{G}_{\mathrm{c}}$ of the retentates crystallized at different temperatures are reported in Table 5. At higher crystallization temperatures, longer induction times and higher $\Delta \mathrm{G}_{\mathrm{c}}$ for all fractions are obtained. At lower crystallization temperatures (higher degrees of undercooling), shorter induction times and lower $\Delta \mathrm{G}_{\mathrm{c}}$ are observed. No trend was observed between the different distillation temperatures, thus distillation conditions appear to have no effect on the energy barrier to nucleation of the retentates obtained.

\section{Microstructure}

No microstructural differences were observed between retentate samples obtained at different distilla-
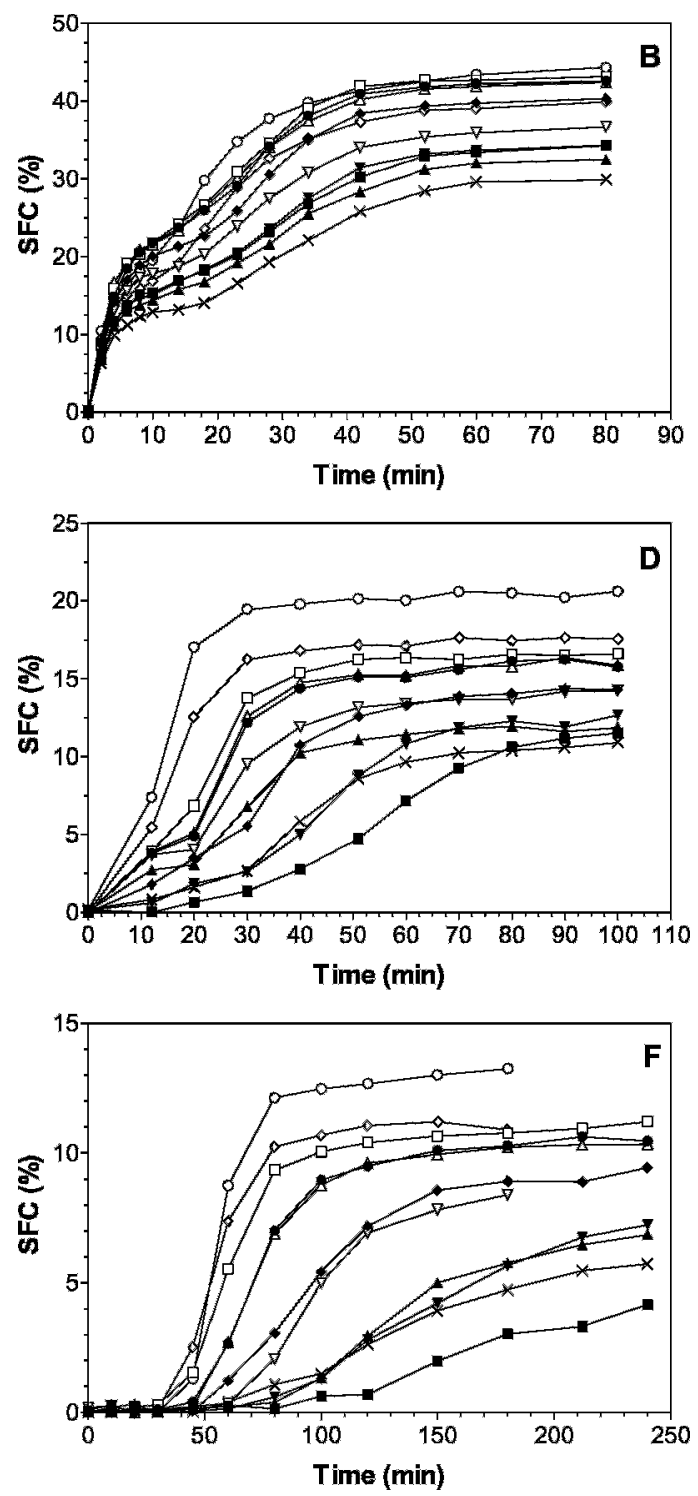

Figure 6. Solid fat content (\%) vs. time (min) during static crystallization at $5(\mathrm{~A}), 10(\mathrm{~B}), 15(\mathrm{C}), 20(\mathrm{D}), 23(\mathrm{E}), 25^{\circ} \mathrm{C}(\mathrm{F})$ of retentates obtained at $125(\mathbf{\square}), 150(\boldsymbol{\Delta}), 175(\nabla), 180(\diamond), 185(\bullet), 190(\square), 195(\triangle), 200(\nabla), 225(\diamond), 250^{\circ} \mathrm{C}(\bigcirc)$, and native milk fat $(\times)$. 

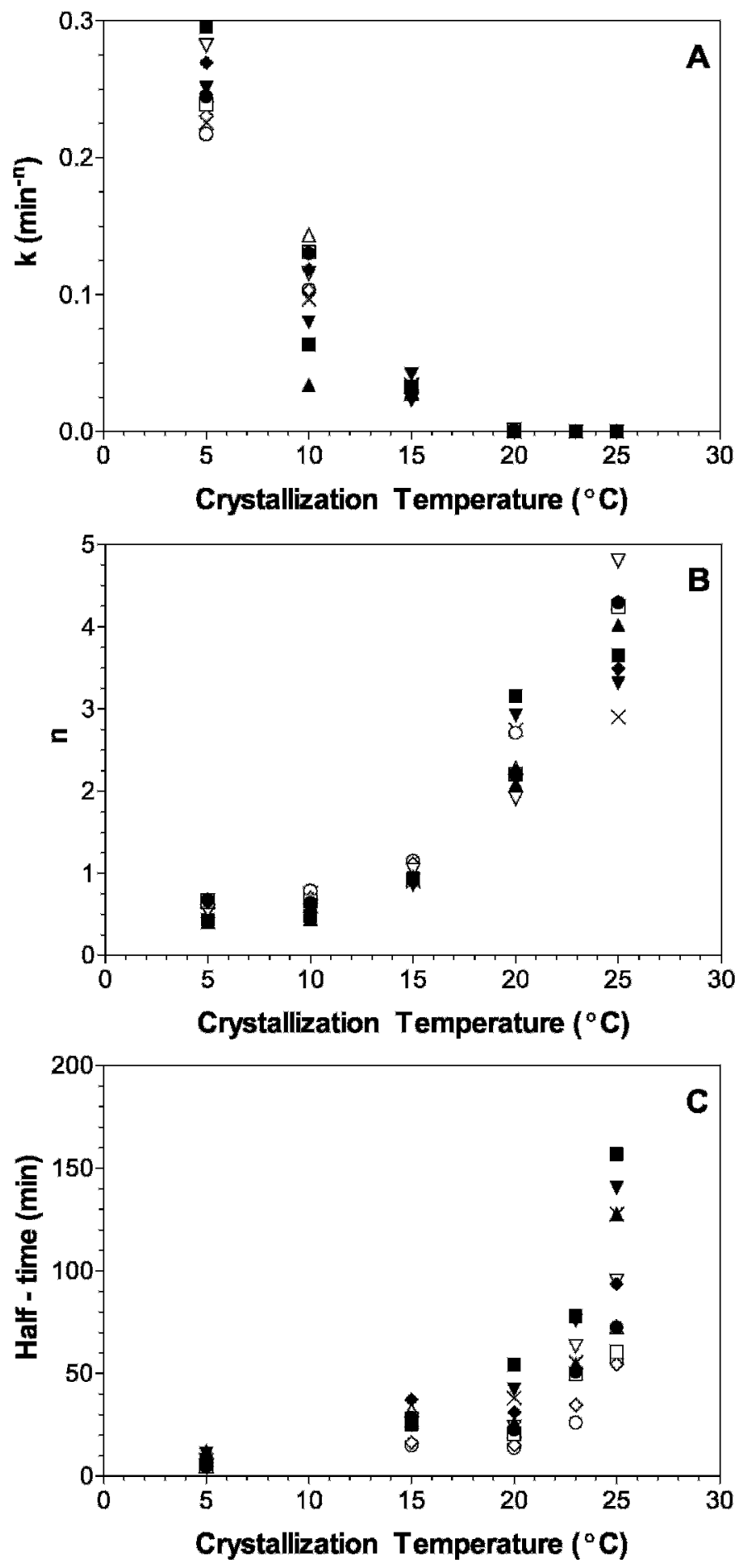

Figure 7. Avrami rate constants $\mathrm{k}$ (A), exponents $n$ (B), and halftimes of crystallization $(\mathrm{C})$ of retentates obtained at $125(\boldsymbol{\square}), 150(\mathbf{\Delta})$, $175(\nabla), 180(\diamond), 185(\bullet), 190(\square), 195(\triangle), 200(\nabla), 225(\diamond), 250^{\circ} \mathrm{C}$ $(\bigcirc)$, and native milk fat $(\times)$. The average dropping point of the retantates was $34.9^{\circ} \mathrm{C}$. tion temperatures. However, large differences were observed for samples above and below $20^{\circ} \mathrm{C}$. Figure 8 shows polarized light micrographs of the retentate (obtained when distilling milk fat at $175^{\circ} \mathrm{C}$ ) crystallized statically for $24 \mathrm{~h}$ at 5 and $25^{\circ} \mathrm{C}$. With a high degree of undercooling (crystallization at $5^{\circ} \mathrm{C}$ ) the nucleation rate is higher due to a lower $\Delta \mathrm{G}_{\mathrm{c}}$, resulting in a granular morphology. Under these conditions, milk fat has been reported to crystallize in the $\alpha$ polymorph (ten Grotenhuis et al., 1999). Conversely, crystallization at higher temperatures $\left(25^{\circ} \mathrm{C}\right)$, in which the nucleation rate is lower, due to a high $\Delta \mathrm{G}_{\mathrm{c}}$, allows for a more orderly crystal growth and formation of spherulitic microstructures. Under these conditions, milk fat crystallizes in the $\beta^{\prime}$ polymorph as reported by ten Grotenhuis et al. (1999).

The morphological differences observed correspond to the change observed in the Avrami parameters above and below $20^{\circ} \mathrm{C}$. From Figure 7 it can be observed that at $5^{\circ} \mathrm{C}$, the value of $n$ is smaller than 1 , whereas at $25^{\circ} \mathrm{C}$ the values of $n$ are 3 or greater. The Avrami exponent is sensitive both to the type of nucleation and dimensionality of growth. Sharples (1966) reported that values of 1 correspond to rod-like growth from instantaneous nuclei, whereas values of 3 or 4 correspond to spherulitic growth from either sporadic or instantaneous nucleation. There is a correspondence between the significance of $n$ and the different morphologies observed in Figure 8. Similar agreement between crystallization kinetics and microstructure was also found by Wright et al. (2001) for milk fat. Our results suggest that the Avrami exponent does provide a phenomenological index of crystallization, which can possibly be used to discern between different mechanisms of crystallization.

\section{Rheology}

The compressive storage modulus $\left(\mathrm{E}^{\prime}\right)$ of retentates obtained at different distillation temperatures are shown in Figure 9. At distillation temperatures of $185^{\circ} \mathrm{C}$ and above, the elastic modulus of retentates is higher, i.e, the material is more elastic, or solidlike, relative to native milk fat. A positive significant correlation $\left(\mathrm{R}^{2}=0.47, P=0.019\right)$ was found between the concentration of long-chain fatty acids and the compressive storage modulus of the studied retentates. Hardness indices of fat networks have been found to positively correlate to the elastic modulus for various lipid systems including milk fat (Narine and Marangoni, 2001). According to this, the higher the distillation temperature, the harder the retentate will be. Other studies have also found that the addition of long-chain fatty acids to milk fat appear to affect its functionality, as 
Table 5. Apparent activation free energies of nucleation $\left(\Delta \mathrm{G}_{\mathrm{c}}\right)$ for retentate obtained at different fractionation temperatures in $\mathrm{kJ} / \mathrm{mol}$.

\begin{tabular}{lllllll}
\hline $\begin{array}{l}\text { Temperature of } \\
\text { fractionation } \\
\left({ }^{\circ} \mathrm{C}\right)\end{array}$ & \multicolumn{5}{c}{ Temperature of crystallization $\left({ }^{\circ} \mathrm{C}\right)$} \\
\cline { 2 - 6 } & 5 & 10 & 15 & 20 & 23 & 25 \\
\hline 125 & 0.34 & 0.49 & 0.79 & 1.48 & 2.42 & 3.66 \\
150 & 0.31 & 0.46 & 0.73 & 1.35 & 2.19 & 3.27 \\
175 & 0.24 & 0.36 & 0.58 & 1.07 & 1.76 & 2.66 \\
180 & 0.30 & 0.44 & 0.69 & 1.27 & 2.04 & 3.02 \\
185 & 0.38 & 0.55 & 0.87 & 1.59 & 2.56 & 3.79 \\
190 & 0.37 & 0.53 & 0.82 & 1.47 & 2.29 & 3.30 \\
195 & 0.30 & 0.44 & 0.69 & 1.25 & 1.98 & 2.91 \\
200 & 0.29 & 0.42 & 0.65 & 1.15 & 1.79 & 2.57 \\
225 & 0.31 & 0.44 & 0.69 & 1.22 & 1.91 & 2.73 \\
250 & 0.24 & 0.34 & 0.54 & 0.98 & 1.56 & 2.28 \\
Native milk fat & 0.37 & 0.52 & 0.80 & 1.38 & 2.08 & 2.90 \\
\hline
\end{tabular}

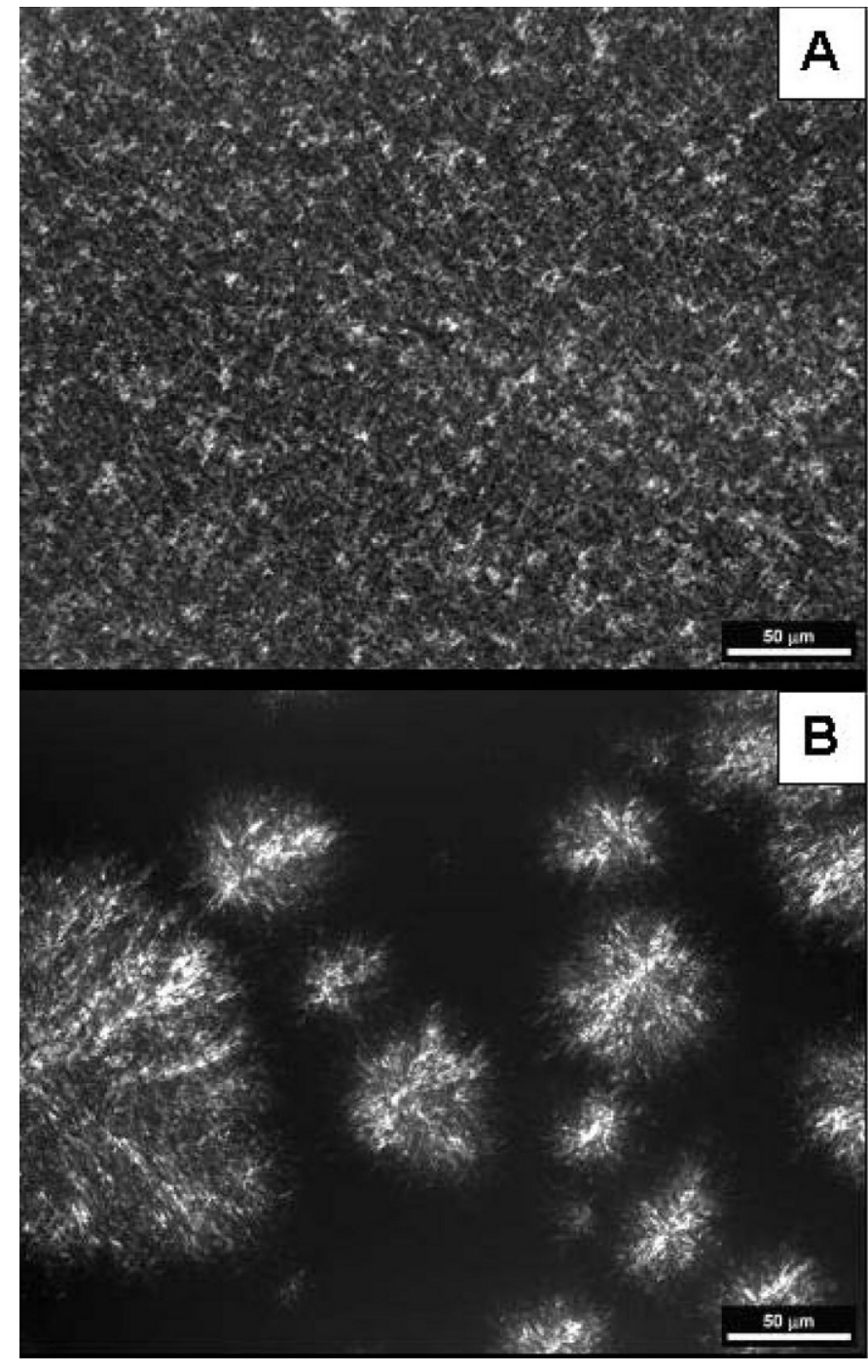

Figure 8. Polarized light micrographs of retentate obtained after distillation at $185^{\circ} \mathrm{C}$ and crystallized statically for $24 \mathrm{~h}$ at 5 (A) and $25^{\circ} \mathrm{C}(\mathrm{B})$ an exponential increase in hardness was reported upon the addition of tripalmitin to butterfat (Fairley et al., 1994).

Short-path distillation offers an alternative method to fractionate milk fat into a heavy fraction (retentate) and a light fraction (distillate) on the basis of volatility. It yields fractions with distinct chemical composition and physical properties. The resulting retentate is enriched in high molecular weight lipid species, while these are depleted in the distillate. On the other hand, the concentration of low molecular fatty acids and saturated species is higher in the distillate relative to that of native milk fat. These changes in chemical composition affect physical characteristics such as melting and rheological behavior, hence functionality of the fractions.

\section{ACKNOWLEDGMENTS}

This research was funded by the Ontario Ministry of Agriculture and Food (Food Research Program). The

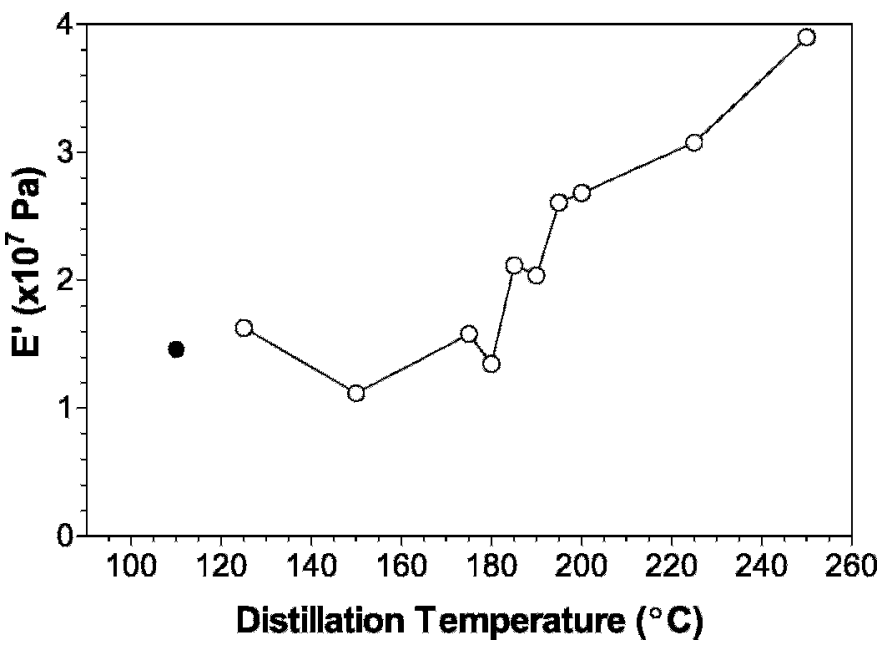

Figure 9. Compressive storage modulus $\left(\mathrm{E}^{\prime}\right)$ of milk fat $(\bullet)$ and retentates $(O)$ obtained at different fractionation temperatures. 
technical assistance of Neena Ghandi is gratefully acknowledged.

\section{REFERENCES}

AOCS. 1983. Official Methods and Recommended Practices of the American Oil Chemists' Society, 4th ed., AOCS Press, Champaign, IL.

Arul, J., A. Boudreau, J. Makhlouf, R. Tardif, and T. Bellavia. 1988. Fractionation of anhydrous milk fat by short-path distillation, J. Am. Oil Chem. Soc. 65:1642-1646.

Avrami, M. 1939. Kinetics of phase change. I. General theory. J. Chem. Phys. 7:1103-1112.

Banks, W. 1991. Chemical and physical properties of milk fat, in utilizations of milkfat, Int. Dairy Fed. Bull. 260:4.

Bannon, C. D., J. D. Craske, and A. D. Hilliker. 1985. Analysis of fatty acid methyl esters with high accuracy and reliability. IV. Fats with fatty acids containing four or more carbon atoms. J. Am. Oil Chem. Soc. 62:1501-1507.

Boudreau, A., and J. Arul. 1993. Cholesterol reduction and fat fractionation technologies of milk fat: An overview. J. Dairy Sci. $76: 1772-1781$

Breitschuh, B. 1998. Continuous dry fractionation of milk fat-application of high shear fields in crystallization and solid-liquid separation. Ph.D. Thesis. Swiss Federal Institute of Technology, Zurich. Pages 1-11.

Deffense, E. 1993. Milk fat fractionation today: A review. J. Am. Oil Chem. Soc. 70:1193-1203.

Dimick, P. S., S. Yella Reddy, and G. R. Ziegler. 1996. Chemical and thermal characteristics of milk-fat fractions isolated by a melt crystallization, J. Am. Oil Chem. Soc. 73:1647-1652.

Fairley, P., J. B. German, and J. M. Krochta. 1994. Phase behavior and mechanical properties of tripalmitin/butterfat mixtures. J. Food Sci. 59:321-337.

German, J. B., and C. J. Dillard. 1998. Fractionated milk fat: Composition, structure, and functional properties, Food Technol. $52: 33-38$.

Hamm, W. 1995. Trends in edible oil fractionation, Trends Food Sci. Technol. 6:121-126.

Hartel, R. W. 2001. Pages 238-240 and 265-270 in Crystallization in Foods. Aspen Publications, Gaithersburg, MD.

Jensen, R. G., A. M. Ferris, and C. J. Lammi-Keefe. 1991. The composition of milk fat. J. Dairy Sci. 74:3228-3243.
Jimenez-Flores, R. 1997. Trends in research for alternate uses of milk fat. J. Dairy Sci. 80:2644-2650.

Kaylegian, K. E., and R. C. Linsay. 1992. Performance of selected milk fat fractions in cold-spreadable butter. J. Dairy Sci. 75:3307-3317.

Krishnamurthy, R., and M. Kellens. 1996. Fractionation and winterization, Pages 301-338 in Edible Oil and Fat Products: Processing Technology, Bailey's Industrial Oil and Fat Products, Y. H. Hui, ed. John Wiley \& Sons Inc. New York.

Marangoni, A. G., and R. W. Lencki. 1998. Ternary phase behavior of milk fat fractions. J. Agric. Food Chem. 46:379-3884.

Marangoni, A. G., 1998. On the use and misuse of the Avrami equation in characterization of kinetics of fat crystallization, J. Am. Oil Chem. Soc. 75:1465-1467.

Narine, S. S., and A. G. Marangoni. 2001. Elastic modulus as an indicator of macroscopic hardness of fat crystal networks, Lebensm. Wiss. Technol. 34:33-40.

Nawar, W. W. 1996. Page 232 in Lipids in Food Chemistry. O. R. Fennema, ed. Marcel Dekker, Inc., New York.

Rajah, K. K. 1994. Milk fat developments. J. Soc. Dairy Technol. 47:81-83.

Rajah, K. K. 1996. Pages 207-210 in Fractionation of Fat in Separation Processes in the Food and Biotechnology Industries: Principles and Applications. A. S. Grandison and M. J. Lewis, eds. Technomics, Lancaster, PA.

Rizvi, S. S. H., and A. R. Bhaskar. 1995. Supercritical fluid processing of milk fat: Fractionation, scale-up, and economics. Food Technol. February:90-100.

Rousseau, D., K. Forestiere, A. R. Hill, and A. G. Marangoni. 1996. Restructuring butterfat through blending and chemical interesterification. 1. Melting behavior and triacylglycerol modifications. J. Am. Oil Chem. Soc. 73:963-972.

Sharples, A. 1996. Overall kinetics of crystallization Pages 44-59 in Introduction to Polymer Crystallization. A. Sharples, ed. Edward Arnol Ltd., London.

ten Grotenhuis, E., G. A. van Aken, K. F. van Malssen, H. Schenk. 1999. Polymorphism of milk fat by differential scanning calorimetry and real-time x-ray powder diffraction. J. Am. Oil Chem. Soc. 76:1031-1039.

van Aken, G. A., and E. ten Grotenhuis, 1999. Composition and crystallization of milk fat fractions. J. Am. Oil Chem Soc. 76:1323-1331.

Wright, A. J., R. W. Hartel, S. S. Narine, and A. G. Marangoni. 2000. The effect of minor components on milk fat crystallization. J. Am. Oil Chem. Soc. 77:463-475. 